\title{
Über die Hydrazone des Fluorenons und seiner Substitutionsproducte
}

\author{
(I. Mittheilung) \\ von \\ Guido Goldschmiedt, c. M. k. Akad. \\ und \\ Franz Schranzhofer.
}

Aus dem chemischen Laboratorium der k. k. deutschen Universität zu Prag.

(Vorgelegt in der Sitzung am 10. October 2895.)

Gelegentlich der Fortsetzung seiner noch unvollendeten Studien über die Ellagsäure hat der Eine von uns sich bemüht, ein Hydrazon dieser Substanz darzustellen; dies gelang auf keine Weise. Da nun nach den bisherigen Kenntnissen über die Ellagsäure angenommen werden muss, dass dieselbe ein Derivat eines Diphenylenketons ist - gibt sie ja doch bekanntlich bei der Reduction mit Zinkstaub glatt Fluoren - so lag es nahe, auch Substitutionsproducte des leicht zugänglichen Fluorenons (Diorthodiphenylenketon) in Bezug auf die Möglichkeit der Bildung von Hydrazonen zu studiren und eventuell zu ermitteln, inwieweit Natur und Stellung der Substituenten hierauf von Einfluss sind.

\section{Fluorenon.}

Durch die schönen Untersuchungen Spiegler's ${ }^{1}$ wissen wir seit Langem, dass dieses Keton mit grösster Leichtigkeit mit Hydroxylamin-Chlorhydrat unter Bildung eines schön krystallisirenden Oxims reagirt, doch ist das Hydrazon desselben

1 Monatshefte für Chemie, V, 195. 
bisher nicht dargestellt worden; wir haben bei dieser Gelegenheit diese Lücke ausgefüllt.

$1 \cdot 5 \mathrm{~g}$ Fluorenon, dargestellt durch Destillation von Phenanthrenchinon über Bleioxyd nach Wittenberg und Meyer ${ }^{1}$ wurden mit etwas mehr als der äquivalenten Menge $(1 \mathrm{~g})$ Phenylhydrazin in alkoholischer Lösung einige Zeit am Wasserbade erwärmt, die Lösung dann mit Essigsäure angesäuert. Beim Erkalten schieden sich schöne gelbe Krystallnadeln aus, welche durch Umkrystallisiren aus Alkohol in glänzenden, bis $1 \mathrm{~cm}$ langen, gestreiften Prismen erhalten werden können, deren nicht mehr erhöhbarer Schmelzpunkt bei $151-151^{\circ} 5$ liegt.

Die Analyse lieferte nachstehende, für Fluorenonhydrazon stimmende Zahlen:

I. $0 \cdot 2723 g$ Substanz gaben $0.8392 g$ Kohlensäure und $0 \cdot 1269 \mathrm{~g}$ Wasser.

II. $0 \cdot 2194 \mathrm{~g}$ Substanz gaben $20 \cdot 3 \mathrm{~cm}^{3}$ Stickstoff ron $22^{\circ}$ und B. $=754 \mathrm{~mm}$.

In 100 Theilen:

\begin{tabular}{|c|c|c|}
\hline \multicolumn{2}{|c|}{ Gefunden } & \multirow{2}{*}{$\begin{array}{r}\text { Berechnet fur } \\
\mathrm{C}_{19} \mathrm{H}_{14} \mathrm{~N}_{2}\end{array}$} \\
\hline I & II & \\
\hline .84 .05 & - & $84 \cdot 44$ \\
\hline $5 \cdot 17$ & - & $5 \cdot 18$ \\
\hline$\ldots$ & $10 \cdot 39$ & $10 \cdot 37$ \\
\hline
\end{tabular}

\section{Chlorsubstitutionsproducte des Fluorenons.}

Es ist bisher nur Ein solches, und zwar das bei $158^{\circ}$ schmelzende, bei der Oxydation von $p$-Dichlorfluoren (Schmelzpunkt $128^{\circ}$ ) von Hodgkinson und Mathews erhaltene, bekannt; die Autoren schreiben diesem Substitutionsproduct des Fluorenons nachstehende Structur zu, und Beilstein bezeichnet es dementsprechend als p-Dichlorfluorenon

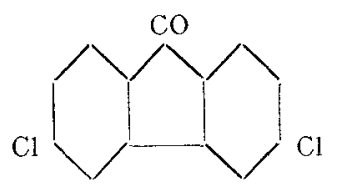

1 Berichte der Deutschen chem. Gesellschaft, XVI, $50 \%$. 
Wir haben nun auch versucht, durch Einwirkung von Chlor auf Fluorenon (aus Phenanthrenchinon) Chlorsubstitutionsproducte darzustellen. $\mathrm{Zu}$ diesem Zwecke wurde das Keton in Chloroform gelöst und trockenes Chlorgas eingeleitet: es wurde keine Chlorwasserstoffentwicklung beobachtet und eine Probe der Lösung hinterliess selbst nach länger andauernder Einwirkung nur reines Diphenylenketon; die Salzsäureentwicklung erfolgt aber sofort, wenn man ein Körnchen Jod in die Lösung wirft.

Nachaem einige Zeit Chlor eingewirkt hatte, wurde das Lösungsmittel abgedunstet, wobei ein dunkelgelber, krystallinischer Rückstand hinterblieb, der nach einmaligem Umkrystallisiren aus Alkohol bei $155-160^{\circ}$ schmilzt, während aus der Mutterlauge hievon zunächst eine bei $105-107^{\circ}$, schliesslich weitere, bei noch niederer Temperatur sich verflüssigende, mit schmierigen Producten verunreinigte Fractionen sich abscheiden.

Durch sehr häufiges systematisches fractionirtes Krystallisiren gelingt es endlich, zwei Substanzen von constantem, durch weitere Krystallisation nicht mehr veränderlichem Schmelzpunkt zu isoliren. Es ist übrigens nicht ausgeschlossen, dass in geringer Menge auch ein um $140-150^{\circ}$ schmelzendes Substitutionsproduct gebildet worden ist.

Das leichter lösliche, in geringerer Menge gebildete Product schmilzt bei $115^{\circ}$ und ist Monochlorfluorenon; das schwerer lösliche, vorwiegend entstandene, verflüssigt sich bei $188-189^{\circ}$ und entspricht einem Dichlorfluorenon, das wir zur Unterscheidung des durch Oxydation von Dichlorfluoren entstehenden, bei $158^{\circ}$ schmelzenden, vorläufig als $\beta$-Dichlorfluorenon bezeichnen wollen.

\section{Monochlorfluorenon.}

Die Substanz vom Schmelzpunkt $115^{\circ}$, von welcher nur eine geringe Menge zur Verfügung stand, krystallisirt aus verdünntem Alkohol, bei langsamer Krystallisation verdünnter Lösungen, in gelben, langen Nadeln, beim Erkalten concentrirter warmer Lösungen in kleinen Nädelchen. 
Die ganz reine Substanz konnte wegen unzureichender Quantität nicht analysirt werden. Eine Fraction, von etwas höherem Schmelzpunkte, die als Erste, aus jener Flüssigkeit ausgefallen war, welche als zweite die scharf bei $115^{\circ}$ schmelzende zur Darstellung des Hydrazons verwendete Substanz abschied, hatte einen Chlorgehalt, der für ein Monochlorfluorenon um ein Geringes zu hoch war.

Die Substanz liefert leicht ein in hellgelben, feinen Nadeln krystallisirendes Hydrazon, wenn man sie in alkoholischer Lösung mit freiem Phenylhydrazin kocht. Das Hydrazon scheidet sich nach dem Ansäuern der Flüssigkeit mit Essigsäure aus der erkalteten Lösung ab. Nach dem Umkrystallisiren schmilzt es bei $139-141^{\circ}$.

$0 \cdot 4011 \mathrm{~g}$ Substanz gaben bei $746 \mathrm{~mm}$ Druck und 1895 $33 \cdot 9 \mathrm{~cm}^{3}$ Stickstoff.

In 100 Theilen:

$$
\text { N...... } \underbrace{\text { Gefunden }}_{9 \cdot 55} \quad \frac{\begin{array}{c}
\text { Berechnet für } \\
\mathrm{C}_{13} \mathrm{H}_{7} \mathrm{Cl}_{2} \mathrm{HC}_{6} \mathrm{H}_{5}
\end{array}}{9 \cdot 19}
$$

\section{P-Dichlorfluorenon.}

Krystallisirt aus Alkohol in schönen, oft sehr langen, glänzenden, gelben Nadeln vom Schmelzpunkte $188-189^{\circ}$; es ist somit bestimmt verschieden von jenem Dichlorsubstitutionsproducte, das durch Oxydation des Dichlorfluorens mit Chromsäure gebildet wird.

$0 \cdot 2804 g$ Substanz lieferten $0 \cdot 3225 \mathrm{~g}$ Chlorsilber.

In 100 Theilen:

$$
\mathrm{Cl} \ldots \ldots \underbrace{\text { Gefunden }}_{28 \cdot 43} \quad \frac{\begin{array}{c}
\text { Berechnet für } \\
\mathrm{C}_{13} \mathrm{H}_{6} \mathrm{Cl}_{2} \mathrm{O}
\end{array}}{28 \cdot 48}
$$

Auch diese Verbindung bildet sehr leicht ein Hydrazon, wenn sie in alkoholischer Lösung einige Zeit mit Phenylhydrazin gekocht wird. Aus der mit Essigsäure angesäuerten 
Flüssigkeit scheidet sich dasselbe in Form von schönen gelben Nadeln ab, die bei $185-186^{\circ}$ schmelzen.

$0.2827 \mathrm{~g}$ Substanz gaben bei $747 \mathrm{~mm}$ Druck und $19^{\circ} 21.8 \mathrm{~cm}^{\prime}$ Stickstoff.

In 100 Theilen:

$$
\mathrm{N} \ldots \ldots \underbrace{\text { Gefunden }}_{8 \cdot 70} \quad \underbrace{\begin{array}{c}
\text { Berechnet fur } \\
\mathrm{C}_{13} \mathrm{H}_{6} \mathrm{Cl}_{2} \mathrm{~N}_{2} \mathrm{HC}_{6} \mathrm{H}_{5}
\end{array}}_{8 \cdot 29}
$$

\section{Bromsubstitutionsproducte des Fluorenons.}

In der Literatur finden sich nachstehende Verbindungen beschrieben.

a-Bromdiphenylenketon, Schmelzpunkt $104^{\circ}$, von Hodgkinson und Matthews ${ }^{1}$ durch Oxydation von Bromfluoren in essigsaurer Lösung dargestellt.

$\beta$-Bromdiphenylenketon, Schmelzpunkt $122^{\circ}$, von Claus und Erler ${ }^{2}$ bei der Destillation von Bromdiphensäure mit Kalkhydrat erhalten.

$\alpha$-Dibromdiphenylenketon, Schmelzpunkt $142^{\circ} 5$, entsteht nach $\mathrm{Holm}^{3}$ bei der Oxydation von $\alpha$-Dibromfluoren mit der theoretischen Menge Chromsäure in Eisessig.

$\beta$-Dibromdiphenylenketon, Schmelzpunkt 197-198 von $\mathrm{Holm}^{4}$ bei der Oxydation desselben Dibromfluorens mit etwas mehr als der theoretischen Quantitat Chromsäureanhydrid, sowie bei der Oxydation von Tribromfluoren erhalten. Ho dg k inson und Matthervs ${ }^{5}$ haben über diesen Gegenstand gleichzeitig mit Holm berichtet; dieselben haben bei der Oxydation von Bibromfluoren aus Steinkohlenfluoren nur die Bildung des bei $198^{\circ}$ schmelzenden Ketons beobachtet und sagen nichts über die Quantität des angewandten Chromsäureanhydrids.

\footnotetext{
1 Journal of the Chemical Society, 1883, p. 165.

2 Berichte der Deutschen chem. Gesellschaft, XIX, $314 y$.

3 Ebenda, XII, 1081.

4 L. c.

5 L. c.
} 
$\gamma$-Dibromfluorenon, Schmelzpunkt $133^{\circ}$, entsteht nach Claus und Erler ${ }^{1}$ bei der Destillation von $\boldsymbol{x}$-Dibromdiphensäure (Schmelzpunkt 245 ${ }^{\circ}$ ) mit Kalkhydrat.

\section{$\beta$-Dibromfluorenon.}

Die Darstellung dieser, wie erwähnt, bereits von Holm auf anderem Wege erhaltenen Verbindung gelingt leicht, wenn man Diphenylenketon mit etwas mehr als der 2 Molekülen entsprechenden Menge Brom übergiesst; es findet eine heftige Reaction statt; nachdem dieselbe beendet, wird am Wasserbade erwärmt, bis kein Bromwasserstoff mehr entweicht, und der zurückbleibende feste Rückstand dann aus Alkohol umkrystallisirt. Man erhält die Substanz in Form von in Alkohol ziemlich schwer löslichen, gelben, sehr feinen Nadeln, die sich am Filter zu einem dichten Filze zusammenlegen. Der Schmelzpunkt dieser Substanz wurde von uns in Übereinstimmung mit $\mathrm{Holm}$ bei $198-199^{\circ}$ beobachtet. Die Analyse dieser Substanz beweist. dass hier ein Dibromfluorenon vorliegt.

$0 \cdot 3039 \mathrm{~g}$ Substanz gaben $0 \cdot 3372 \mathrm{~g}$ Bromsilber.

In 100 Theilen:

$$
\mathrm{Br} \ldots \ldots \underbrace{\text { Gefunden }}_{47 \cdot 18} \quad \frac{\begin{array}{c}
\text { Berechnet } \\
\text { fir } \mathrm{C}_{13} \mathrm{H}_{6} \mathrm{Br}_{2} \mathrm{O}
\end{array}}{4 \% \cdot 33} .
$$

Das Hydrazon des $\beta$-Dibromfluorenons entsteht leicht beim Erwarmen des Ketons mit freiem Phenylhydrazin in alkoholischer Lösung; die mit Essigsäure versetzte Flüssigkeit scheidet beim Erkalten hellgelb gefärbte feine Nadeln des Hydrazons ab, die nach dem Umkrystallisiren aus Alkohol bei raschem Erhitzen sich bei $190^{\circ}$ verflüssigen. Aus verdünnteren Lösungen kann durch langsame Verdunstung das Hydrazon auch in etwas stärkeren, büschelförmig angeordneten Nädelchen von dunklerer Farbe erhalten werden.

$0 \cdot 2453 \mathrm{~g}$ Substanz gaben bei $745 \mathrm{~mm}$ Druck und $19^{\circ} 14 \cdot 8 \mathrm{~cm}^{3}$ Stickstoff. 
In 100 Theilen:

$$
\text { N . . . . . } \underbrace{\text { Gefunden }}_{6.79} \quad \underbrace{\begin{array}{l}
\text { Berechnet } \\
\text { für } \mathrm{C}_{19} \mathrm{H}_{12} \mathrm{Br}_{2} \mathrm{~N}_{2}
\end{array}}_{6 \cdot 5 \frac{1}{t}}
$$

\section{๙-Dibromfluorenon.}

Dibromfluoren vom Schmelzpunkt $165-166^{\circ}$, welches seinerzeit von Barth und Goldschmiedt aus, durch Reduction von Ellagsäure mit Zinkstaub gewonnenem, Fluoren dargestellt worden war, später auch solches, zu dessen Darstellung Fluoren, welches durch die gleiche Reaction aus Fluorenon erhalten worden ist, gedient hatte, wurde nach der Vorschrift von $\mathrm{Holm}^{1}$ mit der theoretischen Menge Chromsäure in essigsaurer Lösung oxydirt. Die Reaction geht ziemlich rasch vor sich und war nur zum Schlusse schwaches Erwärmen erforderlich, um die Chromsäure vollständig zu reduciren. Der grösste Theil des Eisessigs wurde abdestillirt und der Rest dann in Wasser gegossen. Der sich ausscheidende gelbe Niederschlag wurde aus Alkohol, in dem er nicht allzuleicht löslich war, umkrystallisirt. Bald fielen gelbe Nadeln aus, die bei $142-143^{\circ}$ schmolzen, $^{2}$ also jenen Schmelzpunkt zeigten, welchen Hol m für das $\alpha$-Dibromfluorenon angegeben hat.

Diese Übereinstimmung veranlasste uns, im Vertrauen auf die Reinheit der Substanz, von einer Analyse derselben abzusehen, und sofort das Hydrazon darzustellen. Nach zehnstündigem Kochen der alkoholischen Lösung mit Phenylhydrazin, wurde mit Essigsäure versetzt, die sich ausscheidende hell bräunlich-gelb gefä:bte Verbindung aus Alkohol umkrystallisirt, wodurch scheinbar homogene Nadeln, die je nach der Raschheit des Erhitzens bei $134-144^{\circ}$ unscharf schmolzen, erhalten wurden.

Die Analyse I zeigte, dass wir nicht das erwartete Hydrazon in Händen hatten.

1 B. XVI, 1081.

* Es sei erwähnt, dass, wenn bei der Oxydation sofort gekocht wird, der Schmelzpunkt des Productes stets höher liegt. 
Es wurde in Folge dessen eine andere Portion des bei $142-143^{\circ}$ schmelzenden Productes, ohne Anwendung eines Lösungsmittels zehn Stunden mit überschüssigem Phenylhydrazin am Wasserbade erwärmt, und das Reactionsproduct, wie oben, abgeschieden und aus Alkohol umkrystallisirt. Das Aussehen der so erhaltenen Verbindung war ähnlich dem des zuerst beschriebenen Productes, nur war es vielleicht etwas dunkler gefärbt. Auch das Verhalten im Schmelzpunktbestimmungsapparate war dasselbe.

Die Analyse II liefert den Nachweis, dass die Zusammensetzung dieselbe war, wie bei der zuerst dargestellten Substanz.

Hierauf wurden die von den Analysen I und II erübrigten Reste obiger zwei Präparate vereinigt und mit überschüssigem Phenylhydrazin unter Rückfluss zwei Stunden im Sieden erhalten und dann in üblicherWeise abgeschieden und umkrystallisirt. Auch diese energische Behandlung führte zu keiner Veränderung im Verhalten und in der Zusammensetzung (Analyse III und IV) der Substanz.

Als das vermeintliche $\alpha$-Dibromfluorenon mit salzsaurem Phenylhydrazin und essigsaurem Natrium durch mehrere Stunden gekocht wurde, entstand ein Product, das nach dem Umkrystallisiren beim Erhitzen sich ebenso verhielt, wie die früher beschriebenen, und zwar gilt dies von der zuerst ausfallenden Fraction, wie von der zweiten. Dieses Präparat war etwas heller gefärbt und besser krystallisirt, anscheinend auch homogen. (Analyse V und VI).

I. $0 \cdot 6126 \mathrm{~g}$ Substanz gaben bei $746 \mathrm{~mm}$ Druck und $20^{\circ}$ $15 \cdot 8 \mathrm{~cm}^{3}$ Stickstoff.

II. $0 \cdot 6090 \mathrm{~g}$ Substanz gaben bei $740 \mathrm{~mm}$ Druck und $17^{\circ}$ $14 \cdot 8 \mathrm{~cm}^{3}$ Stickstoff,

III. $0 \cdot 4815 \mathrm{~g}$ Substanz gaben bei $738 m m$ Druck und $18^{\circ}$ $11 \cdot 2 \mathrm{~cm}^{3}$ Stickstoff.

IV. $0 \cdot 2634 \mathrm{~g}$ Substanz gaben $0 \cdot 2738 \mathrm{~g}$ Bromsilber.

V. $0.5553 \mathrm{~g}$ Substanz gaben bei $736 \mathrm{~mm}$ Druck und $19^{\circ}$ $13.5 \mathrm{~cm}^{3}$ Stickstoff.

VI. $0.2302 g$ Substanz gaben $0.4186 g$ Kohlensäure und $0.0572 \mathrm{~g}$ Wasser. 
In 100 'Theilen:

\begin{tabular}{|c|c|c|c|c|c|c|c|}
\hline & \multicolumn{6}{|c|}{ Gefunden } & \multirow{2}{*}{$\begin{array}{l}\text { Berechnet für } \\
\mathrm{C}_{19} \mathrm{H}_{13} \mathrm{Br}_{2} \mathrm{~N}_{2}\end{array}$} \\
\hline & I & II & III & IV & $\bar{r}$ & VI & \\
\hline C. & - & - & - & $\ldots$ & $\ldots$ & $49 \cdot 59$ & $53 \cdot 27$ \\
\hline $\mathrm{H} \ldots \ldots$ & - & - & - & - & 一 & $2 \cdot 76$ & $2 \cdot 80$ \\
\hline $\mathrm{Br}$ & - & - & 一 & $44 \cdot 22$ & - & - & $37 \cdot 38$ \\
\hline$N \ldots \ldots$ & $2 \cdot 89$ & $2 \cdot 7$ & $2 \cdot 60$ & - & $2 \cdot 70$ & - & $6 \cdot 54$ \\
\hline
\end{tabular}

Es geht aus obiger Zusammenstellung mit Sicherheit hervor, dass die zu unseren Analysen verwendeten Substanzen nicht das Hydrazon eines Dibromfluorenons sein können. DerUmstand, dass die Substanz homogen aussah und, trotz verschiedener Art der Darstellung und wiederholter Krystallisation, stets die gleiche Zusammensetzung hatte, machte es uns schwer, zu glauben, dass dieselbe aus einem Gemenge bestehe.

Wir hielten es unter allen Umständen zunächst für nothwendig, uns nachträglich durch Analyse von der Beschaffenheit unseres Ausgangsproductes, das heisst des vermeintlichen x-Dibromdiphenylenketons zu überzeugen.

$0 \cdot 4200 \mathrm{~g}$ Substanz gaben $0 \cdot 4780 \mathrm{~g}$ Bromsilber.

In 100 Theilen;

\begin{tabular}{|c|c|c|c|}
\hline \multirow[b]{2}{*}{ Gefunden } & \multicolumn{3}{|c|}{ Berechnet fur } \\
\hline & $\mathrm{C}_{13} \mathrm{H}_{6} \mathrm{Br}_{2} \mathrm{O}$ & $\mathrm{C}_{13} \mathrm{H}_{8} \mathrm{Br}_{2}$ & $\mathrm{C}_{13} \mathrm{H}_{6} \mathrm{Br}_{2} \mathrm{O}+2 \mathrm{C}_{13} \mathrm{H}_{6} \mathrm{Br}_{2}$ \\
\hline $48 \cdot 2 c$ & $47 \cdot 34$ & $49 \cdot 44$ & $48 \cdot 17$ \\
\hline
\end{tabular}

Aus obiger Zusammenstellung ist ersichtlich, dass der Bromgehalt des Präparates zwischen jenem liegt, welcher dem Dibromfluoren und jenem, der dem Dibromfluorenon zukömmt und jenem entspricht, welcher einem Gemische von 1 Moleküle Keton mit 2 Molekülen Dibromfluoren zukommen würde.

Durch sehr häufiges Umkrystallisiren des bei $143-144^{\circ}$ schmelzenden Präparates aus Alkohol gelingt es, eine in gelben Nadeln krystallisirende und bei $197^{\circ}$ schmelzende Verbindung, d. i. $\beta$-Dibromfluorenon in nicht unbeträchtlicher Menge $z \mathrm{u}$ isoliren; ausserdem wurden Fractionen von tieferem Schmelzpunkte 
erhalten, unter andern unscharf bei circa $160^{\circ}$ schmelzende, nicht mehr gelb gefärbte Nadeln, sondern Blättchen, die jenen des Dibromfluorens auffallend ähnlich sahen, und weiter Fractionen, deren unscharfer Schmelzpunkt zwischen $136^{\circ}$ und $146^{\circ}$ lag, die also gewiss nicht rein waren, ihrer geringen Menge wegen aber nicht weiter verarbeitet werden konnten.

Wir halten es nicht für unwahrscheinlich, dass hier eine moleculare Verbindung von Dibromfluoren mit einem Dibromfluorenon vorliegt, welche auch in der Verbindung mit Phenylhydrazin erhalten bleibt. Für diese Ansicht, die wir mit aller Reserve aussprechen, spricht die Homogenität der bei $143^{\circ}$ schmelzenden Substanz und des daraus erhaltenen Hydrazinproductes. ${ }^{1} \mathrm{Ob}$ bei dem häufigen Umkrystallisiren und der hiebei erfolgenden Zerlegung der vermutheten molecularen Verbindung eine Umlagerung des von Holm beschriebenen $\alpha$-Dibromfluorenons in die $\beta$-Verbindung stattfindet, ob in den gelben Fractionen, deren Schmelzpunkt dem von Holm für diese Verbindung angegebenen nahe liegt, diese in noch unreinem Zustande vorliegt, ob dieses $\alpha$-Dibromfluorenon überhaupt existirt, was wir durchaus nicht in Abrede stellen können, sind Fragen, die wir vorläufig nicht beantworten konnten, da uns Materiale für neue Versuche fehlte.

Schon vor der Ausführung der eben beschriebenen Versuche hatten wir Dibromfluoren vom Schmelzpunkte $165-166^{\circ}$ auch mit einem Überschusse von Chromsäure oxydirt, - und zwar wurden $10.5,20.6$ und 31.8 Procent mehr als die theoretisch erforderliche Menge angewendet - - um auf diesem Wege nach Holm's Angabe $z u$ dem von uns durch directe Substitution erhaltenen $\mathrm{F}$-Dibromfluorenon $\mathrm{zu}$ gelangen. In keinem Falle wurde hiebei in glatter Reaction das Ziel erreicht.
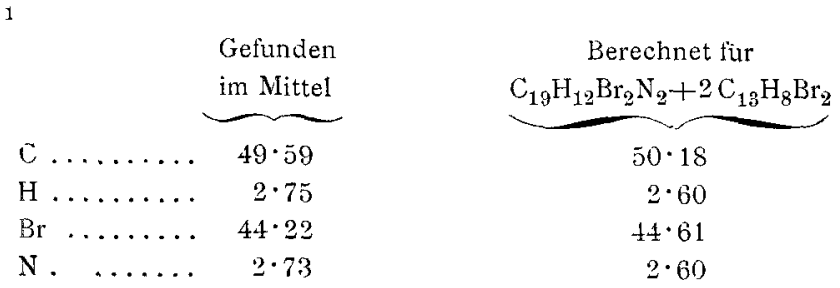
Obzwar das erwartete Product stets enstanden war und durch Krystallisation aus Alkohol in reinem Zustande abgeschieden werden konnte, so hatten sich ausserdem stets in nicht unbeträchtlicher Menge auch Substanzen von tieferem Schmelzpunkte gebildet.

DieIdentität dieses $\beta$-Fluorenons mit dem durch Substitution bereiteten wurde durch dessen Schmelzpunkt und durch Darstellung des Hydrazons, welches wie das des Letzteren be $190-191^{\circ}$ schmolz, sicher gestellt. Nach unseren Erfahrungen hat demnach die Menge der zur Oxydation des Dibromfluorens verwendeten Chromsäure insoferne keinen wesentlichen Einfluss auf den Verlauf der Reaction, als aus dem Reactionsproducte unter allen Umständen das höher schmelzende $\beta$-Dibromfluorenon erhalten werden kann. Weitere Versuche in dieser Richtung sind jedoch zur endgültigen Aufklärung des Vorganges noch erforderlich.

\section{$\beta$-Bromfluorenon und $\gamma$-Dibromfluorenon.}

Diese beiden Substanzen sind von Claus und Erler ${ }^{1}$ aus Mono-, beziehungsweise Dibromdiphensäure dargestellt worden; wir haben $z \mathrm{u}$ ihrer Bereitung die Vorschrift der genannten Autoren befolgt.

Es wurde reine Diphensäure aus Phenanthren nach Fittig² dargestellt und dann nach Claus und Erler bromirt. Einem Moleküle Diphensäure wurden vier Atome Brom zugesetzt und das Gemisch in Einschmelzröhren durch drei Stunden auf $200-210^{\circ}$ erhitzt. Es sind dies jene Bedingungen, unter welchen, nach Claus und Erler, nur Mono- und Dibromdiphensäure ohne Nebenproducte entstehen. Beim Öffnen der Röhren zeigte sich sehr hoher Druck und es entwich eine beträchtliche Menge Bromwasserstoff.

Nachdem das röthlich gefärbte, in festen Krusten erhaltene Reactionsproduct, nach Vorschrift der genannten Autoren, mit kaltem Chloroform von den färbenden Verunreinigungen befreit worden war, wurde versucht, den Angaben Claus' und Erler's

1 Ber. XIX, 3149.

2 Liebig's Ann., 193, 11 . 
folgend, die beiden entstandenen bromirten Diphensäuren durch die auffallend verschiedene Löslichkeit ihrer Barytsalze in Wasser zu trennen. Es wurde daher das Gemenge der Säuren mit einem geringen Überschusse von bei gewöhnlicher Temperatur gesättigtem Barytwasser durch eine halbe Stunde, ohne zu kochen, erwärmt, hierauf filtrirt und das in Wasser leicht lösliche Barytsalz mit Salzsäure zersetzt.

Es zeigte sich jedoch dabei, dass durch Zersetzung des in Wasser leicht löslichen Barytsalzes nicht Dibromdiphensäure, wie Claus und Erler angeben, sondern Monobromdiphensäure abgeschieden wird. Der Schmelzpunkt der erhaltenen weissen Krystalle, der nach wiederholtem Umkrystallisiren aus Eisessig sich nicht weiter erhöhte, lag bei $236-237^{\circ}$, was mit der Angabe von $\mathrm{Cla}$ us und Erler für den Schmelzpunkt der Monobromdiphensäure, $235-236^{\circ}$, nahe übereinstimmt. Die Analyse ergab auch den für Monobromdiphensäure gültigen Bromgehalt. $0.3041 \mathrm{~g}$ bei $120^{\circ}$ getrockneter Substanz, die dabei nichts an Gewicht verlor, ergaben $0 \cdot 17+3$ Bromsilber.

In 100 Theilen:

$\mathrm{Br} \ldots \ldots 24 \cdot 39 \quad \frac{\begin{array}{c}\text { Berechtet } \\ \text { fur } \mathrm{C}_{14} \mathrm{H}_{9} \mathrm{BrO}_{1}\end{array}}{24 \cdot 92}$

Dass bezüglich der Löslichkeit der Barytsalze in den Angaben von $\mathrm{Claus}$ und Erler eine Verwechslung vorliegen müsse, geht auch aus den Versuchen hervor, die wir mit der abgeschiedenen reinen Monobromdiphensäure ausgeführt haben. Diese löst sich nämlich in kaltem Barytwasser leicht und vollständig auf. Erwärmt man die Lösung, so scheidet sich eine reichliche Krystallisation des Baryumsalzes ab, die sich beim Erkalten der Flüssigkeit nicht wieder löst; aus der Lösung des Salzes und auch aus den durch Erwärmen zur Abscheidung gebrachten Krystallen, wird durch Salzsäure die Monobromdiphensäure vom richtigen Schmelzpunkte wiedergewonnen.

Die Zerlegung des von Barytwasser in der Wärme nicht gelösten Antheiles durch Salzsäure führte zu einem gelblichen Producte, das sich durch Umkrystallisiren in Eisessig leicht in 
zwei wesentlich verschiedene Körper zerlegen liess. Aus der Eisessiglösung fielen zuerst weisse, körnige Krystalle, welche nach mehrmaligem Umkrystallisiren bei $245-246^{\circ}$ schmolzen, hierauf gelbe Nadeln vom Schmelzpunkte 256-262 ${ }^{\circ}$, von welchen später die Rede sein wird.

Die weissen krystallinischen Körner vom Schmelzpunkte $245-246^{\circ}$ sind in Natriumcarbonat unter Kohlensäureentwicklung löslich.

$0.3074 \mathrm{~g}$ der bei $130^{\circ}$ getrockneten Substanz ergaben $0.2879 g$

Bromsilber.

In 100 Theilen:

$$
\text { Br.... } \underbrace{\text { Gefunden }}_{39 \cdot 85} \frac{\begin{array}{c}
\text { Berechnet } \\
\text { fur } \mathrm{C}_{11} \mathrm{H}_{8} \mathrm{Br}_{2} \mathrm{O}_{4}
\end{array}}{40 \cdot 00}
$$

Die untersuchte Substanz ist somit Dibromdiphensäure.

Einzelne Krystallisationen, welche bei der Reinigung der Dibromdiphensäure durch Eisessig erhalten worden waren, hatten einen etwas niederen Schmelzpunkt; dieselben wurden mit kaltem Barytwasser behandelt, wodurch ein Theil in Lösung ging, der sich nach der Abscheidung durch Salzsäure als Monobromdiphensäure erwies; die Substanz hatte den richtigen Schmelzpunkt und löste sich leicht in kaltem Barytwasser. Es ist wohl eine Folge des Erwärmens, welches bei der Trennung der beiden Säuren durch Barytwasser stattgefunden hat, dass nicht unbeträchtliche Mengen der Monobromsäure in dem schwerlöslichen Rückstand zurückgeblieben waren. Übrigens löst sich Dibromdiphensäure in kaltem Barytwasser auch recht merklich, und wenn die Lösungen nicht gar zu verdünnt sind, bilden sich auch hier beim Kochen krystallinische Niederschläge.

Zur vollständigen Reinigung wurde schliesslich der Rest der Säure in kaltem Alkohol gelöst, die Lösung erwärmt und mit heissem Wasser versetzt, bis eine geringe Trübung entstand; beim Erkalten schied sich dann die Säure in schönen, glitzernden, kleinen Nadeln aus, deren Schmelzpunkt bei $245^{\circ}$ bis $246^{\circ}$ lag. 
In der wiederholt citirten Abhandlung von Claus und Erler heisst es: "Bei der trockenen Destillation mit Kalkhydrat entstehen, wie zu erwarten war, aus der einfach gebromten sowohl, wie aus der zweifach gebromten Diphensäure als fassbare Producte nur die entsprechenden gebromten Diphenylenketone. « Auf derselben Seite der Abhandlung heisst es weiter: "Zu seiner Darstellung (i. e. Monobromdiphenylenketon) durch Destillation mit Kalk muss vorsichtig verfahren werden « und auf der nächstfolgenden: $\gg$ Die Dibromdiphensäure bildet bei der Destillation mit Kalkhydrat.... Dibromdiphenylenketon .

In der den gleichen Gegenstand behandelnden Dissertation von Erler ${ }^{1}$ lautet die Überschrift zu dem diesbezüglichen $\mathrm{Ab}$ schnitte "Einwirkung von Ätzkalk auf Monobromdiphensäure «, beziehungsweise »Dibromdiphensäure «, auch im Texte ist stets nur von » Kalk « oder »Ätzkalk « die Rede.

Obgleich nun aus diesen sich theilweise widersprechenden Angaben nicht mit Sicherheit entnommen werden konnte, ob die mehrfach genannten Autoren Ätzkalk oder Kalkhydrat angewendet haben, haben wir mit Rücksicht auf die von Fittig und Ostermayer ${ }^{2}$ und von Anschütz und Schulz mitgetheilten Erfahrungen, wonach Diphensäure beim Erhitzen mit Kalk vorzugsweise in Diphenylenketon, mit gelöschtem Kalk aber hauptsächlich in Diphenyl ühergeführt werde, angenommen, dass in der Abhandlung von Claus und Erler ein Versehen in der Ausdrucksweise unterlaufen sei und dass thatsächlich Ätzkalk verwendet worden ist.

Wir haben dementsprechend zunächst Dibromdiphensäure mit Ätzkalk in der von Claus und Erler beschriebenen Weise destillirt und können bezüglich des Ganges der Operation und der Ausbeute, die recht unbefriedigend war, die Beobachtungen dieser Forscher vollauf bestätigen, doch haben wir durch wiederholte Krystallisation des Destillates aus Alkohol als Hauptproduct eine in gelben Nadeln anschiessende Substanz erhalten,

1 Über Bromdiphensäuren und einige Derivate derselben. Freiburg i. B., 1885.

2 Liebig's Annalen, $166,372$.

: Ebendaselbst, 196, 48. 
die schliesslich bei $196-197^{\circ}$ schmolz. Da wir vermutheten, dass sich in unserem Falle das bei $198-199^{\circ}$ schmelzende $\beta$-Dibromfluorenon gebildet habe, haben wir die geringe verfügbare Quantität auf gewöhnliche Weise in das Hydrazon verwandelt, und an diesem den Schmelzpunkt $186^{\circ}$ beobachtet ( $\beta$-Dibromfluorenonhydrazon $190-191^{\circ}$ ).

Eine Stickstoff bestimmung dieser Substanz zeigte, dass das vermuthete Hydrazon vorliegt.

$0.1974 \mathrm{~g}$ der bei $100^{\circ}$ getrockneten Substanz gaben $11.6 \mathrm{~cm}^{3}$ feuchten Stickstoff bei $20^{\circ}$ und $7+5 m m$ Druck.

In 100 Theilen:

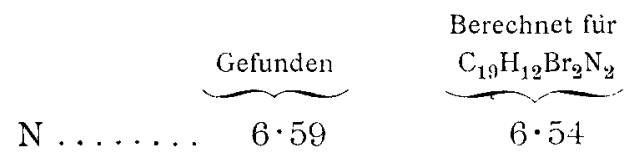

Die leichter löslichen kleinen Fractionen des Destillates hatten einen um $130^{\circ}$ liegenden Schmelzpunkt; wir haben aus denselben ein bei circa $139^{\circ}$ schmelzendes Hydrazon dargestellt, dessen Menge aber zur Analyse unzureichend war. Ob hier das bei $133^{\circ}$ schmelzende Product, welches von Claus und Erler als $\gamma$-Dibromdiphenylenketon bezeichnet wurde, vorliegt, sind wir zu entscheiden natürlich nicht im Stande, müssen es aber für wahrscheinlich halten. Ein kleiner Rest der Dibromdiphensäure wurde nun auch mit gelöschtem Kalk erhitzt und festgestellt, dass auch hier das hochschmelzende $\beta$-Dibromfluorenon gebildet worden war.

In gleicher Weise wurde auch die Monobromdiphensäure mit Kalk destillirt; die Operation, welche nur einmal mit einer geringen Menge Säure durchgeführt werden konnte, lieferte eine recht kleine Ausbeute; das nur einmal aus verdünntem Alkohol umkrystallisirte gelbe Destillat lieferte bei $120^{\circ}$ schmelzende gelbe Krystallblättchen, welche wohl identisch sind mit dem von Claus und Erler beschriebenen, bei $122^{\circ}$ schmelzenden, daher wohl reinerem. $\beta$-Bromdiphenylenketon. Nebenbei scheint auch etwas Fluoren entstanden zu sein. Zur Analyse reichte das Präparat nicht aus, doch wurde auch bei diesem Keton festgestellt, dass es mit Phenylhydrazin ein 
Hydrazon zu bilden fähig ist, das bei $100^{\circ}$ bei raschem Erhitzen schmilzt.

\section{j-Dibromfluorenon.}

Bei der Darstellung der bromirten Diphensäuren war, wie bereits erwähnt, nebst diesen noch ein gelber, bei $262^{\circ}$ unter vorausgehendem Sintern schmelzender Körper in nicht unbeträchtlicher Menge entstanden, welcher nicht mit dem nach Claus und Erler bei $256^{\circ}$ schmelzenden Monobromdiphensäuredibromid identisch sein konnte. Vor Allem unterscheidet er sich durch seine gelbe Farbe von diesem, das farblos ist, ferner ist er unlöslich in kohlensaurem Natron. Durch nochmaliges Umkrystallisiren wurde der Schmelzpunkt dieser Substanz nicht erhöht; sie stellt hellgelbe, weiche, wollige Nadeln dar, die sublimirbar sind.

Die Elementaranalyse derselben ergab, dass auch dieser Körper ein Dibromfluorenon ist, welches wir zur Unterscheidung von den Isomeren als ò-Dibromfluorenon bezeichnen.

I. $0.2849 \mathrm{~g}$ der bei $100^{\circ}$ getrockneten Substanz gaben $0.0590 \mathrm{~g}$ Wasser und $0.4815 \mathrm{~g}$ Kohlensäure.

II. $0.3046 \mathrm{~g}$ der bei $120^{\circ}$ getrockneten Substanz ergaben $0 \cdot 3384 \mathrm{~g}$ Bromsilber.

In 100 Theilen:

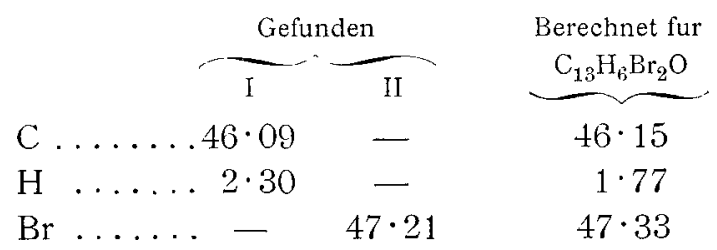

Dem in heissem Alkohol noch nicht völlig gelösten $\delta$-Dibromdiphenylenketon wurde ein Überschuss von Phenylhydrazin zugesetzt, worauf sich Alles löste. Während des Kochens fielen dann orangegelbe, breite, glänzende Nadeln aus. Es wurde mit Eisessig angesäuert, nach dem Erkalten abgesaugt und die Krystalle mit kaltem Alkohol gewaschen.

Dieselben sintern bei $250^{\circ}$ und schmelzen bei $252^{\circ}$ unter heftiger Gasentwicklung. 
Es wurde versucht, dieselben in Alkohol oder Eisessig zu lösen, doch sind dieselben in beiden Lösungsmitteln äusserst schwer löslich, wesshalb sie durch Auskochen mit Eisessig gereinigt wurden. Der Schmelzpunkt veränderte sich durch das Auskochen nicht.

Leicht löslich sind dieselben in Aceton, aus dem sie rein gelb erhalten werden können.

$0.3094 \mathrm{~g}$ der bei $120^{\circ}$ getrockneten Substanz gaben $18.5 \mathrm{~cm}^{3}$ feuchten Stickstoff bei $20^{\circ}$ und $736 \mathrm{~mm}$ Druck.

In 100 Theilen:

$$
\text { N ...... } \underbrace{\text { Gefunden }}_{6 \cdot 62} \underbrace{\begin{array}{c}
\text { Berechnet für } \\
\mathrm{C}_{19} \mathrm{H}_{12} \mathrm{~N}_{2} \mathrm{Br}_{2}
\end{array}}_{6 \cdot 54}
$$

\section{Nitroverbindungen des Fluorenons.}

Nachstehende Nitroproducte sind bekannt:

Nitrofluorenon von Schulz ${ }^{1}$ durch Einwirkung abgekühlter rauchender Salpetersäure auf das Keton dargestellt; es schmilzt bei $220^{\circ}$. Die Structur desselben muss wohl durch nachstehende Formel ausgedrückt werden:

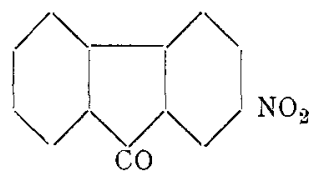

Dinitrofluorenon durch Erwärmen des Ketons mit rauchender Salpetersäure und durch Oxydation von Dinitrofluoren, durch Nitrirung von Fluorenalkohol und durch Oxydation der Dinitrodiphenylglycolsäure von demselben Autor dargestellt. Es schmilzt bei $290^{\circ}$ und seine Structur entspricht der Formel

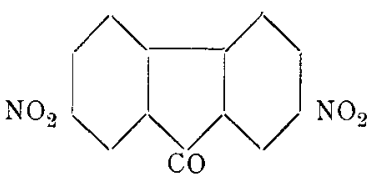

1 Liebig's Annalen, 203. 103. 
In der Absicht, das Schulz'sche Mononitrofluorenon darzustellen, wurde Fluorenon in durch kaltes Wasser gekühlte rauchende Salpetersäure eingetragen. Nach erfolgter Lösung wurde in Wasser gegossen und die abgeschiedenen Nitroproducte abfiltrirt und gewaschen, dann aus Alkohol umkrystallisirt, beziehungsweise mit Alkohol ausgekocht. Wir konnten zwei Verbindungen isoliren, von denen eine bei $290^{\circ} \mathrm{schmolz}$, die andere bei $218^{\circ}$. Wir glaubten somit das bekannte Di-, beziehungsweise Mononitrofluorenon in Händen zu haben.

Für die erstere Verbindung war dies auch zutreffend, während die zweite nicht Mono-, sondern ein isomeres Dinitrofluorenon gewesen ist.

Wir bezeichnen zu ihrer Unterscheidung das bekannte als $\alpha$-Dinitrofluorenon, das neue Isomere als $\beta$-Dinitrofluorenon. Ein ähnliches Resultat erhielten wir, als wir in (nicht rothe) Salpetersäure (spec. Gew. 1.5), welche auf $-10^{\circ}$ abgekühlt war, fein zerriebenes Fluorenon allmälig eintrugen und durch Rühren die Lösung unter fortwährender Abkühlung beförderten. Die Flüssigkeit wurde in Wasser gegossen, der entstandene Niederschlag abfiltrirt und gewaschen. Durch fractionirte Krystallisation aus Alkohol und Eisessig konnte aber ausser den beiden obengenannten Verbindungen auch das bekannte Mononitrofluorenon in geringer Menge abgeschieden werden.

\section{Mononitrofluorenon.}

Diese Substanz krystallisirt, wie bereits Schulz beobachtete, je nach der Concentration der Lösung in Alkohol, in gelben Nadeln oder Blättchen; wir können überhaupt die Beohachtungen Schulz's in jeder Richtung bestätigen.

$0 \cdot 2155 \mathrm{~g}$ Substanz gaben bei $746 \mathrm{~mm}$ Druck und $25^{\circ} 12 \cdot 3 \mathrm{~cm}^{3}$ feuchten Stickstoff.

In 100 Theilen:

$$
\text { N . . . . . } \underbrace{\text { Gefunden }}_{6.23} \quad \underbrace{\begin{array}{c}
\text { Berechnet für } \\
\mathrm{C}_{13} \mathrm{H}_{7} \mathrm{O}\left(\mathrm{NO}_{2}\right)
\end{array}}_{6.22}
$$


Löst man die Substanz in kochendem Eisessig und fügt Phenylhydrazin hinzu, so färbt sich die Lösung intensiv roth; kocht man durch einige Minuten, so fällt beim Erkalten ein dichter, lebhaft rother Niederschlag, Nitrofluorenonhydrazon, aus, der getrocknet ziegelroth ist und unter dem Mikroskope als zu kugeligen Aggregaten zusammengelagerte, ausserordentlich feine, kurze Nädelchen erscheint. Die Substanz schmilzt, rasch erhitzt, bei $210-214^{\circ}$ unter Gasentwicklung; sie ist in Alkohol und Eisessig sehr schwer löslich, leicht in Benzol, Aceton und Chloroform. Von einer Analyse des Hyurazons mussten wir wegen Mangel an Material absehen.

$$
\alpha \text {-Dinitrofluorenon. }
$$

Die Eigenschaften dieser Verbindung fanden wir in vollkommener Übereinstimmung mit den Angaben von Schultz.

Die Zusammensetzung derselben wurde durch Analyse controllirt.

$0 \cdot 1635 g$ Substanz gaben $0.3438 g$ Kohlensäure und $0.0376 g$ Wasser.

In 100 Theilen:

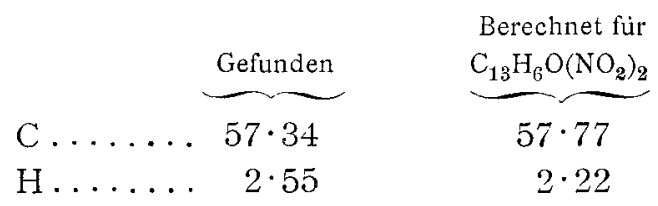

Das Keton wurde in Eisessig gelöst, in welchem es selbst in derKochhitze ziemlich schwer löslich war und Phenylhydrazin zugegeben. Die Flüssigkeit färbte sich tiefroth und es schieden sich sofort dunkel pompejanisch-rothe glitzernde Nadeln ab, die bei raschem Erhitzen bei $257-258^{\circ}$ unter stürmischer Zersetzung schmolzen. Kocht man das Präparat mit ungenügenden Mengen Eisessig aus, so schmilzt das ungelöst gebliebene, wie das aus der Lösung abgeschiedene bei der oben angegebenen Temperatur. Das Hydrazon ist in Alkohol sehr schwer löslich, in Eisessig gleichfalls, doch etwas besser. Aus Eisessig wird es stets ganz homogen in Form gekrümmter langer Nadeln erhalten, aus Alkohol in Nadeln oder Blättchen. 
I. $0.2462 \mathrm{~g}$ bei $100^{\circ}$ getrockneter Substanz gaben $0.5722 \mathrm{~g}$ Kohlensäure und $0.0823 g$ Wasser.

II. $0 \cdot 1994 \mathrm{~g}$ bei $100^{\circ}$ getrockneter Substanz gaben $27 \cdot 30 \mathrm{~cm}^{3}$ Stickstoff bei $756 \mathrm{~mm}$ Druck und $22 \cdot 5^{\circ}$.

In 100 Theilen:

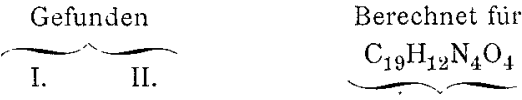

$$
\begin{aligned}
& \text { C....663.33- } 63 \cdot 33 \\
& \text { H.... 3.72 - } 3 \cdot 33 \\
& \text { N... }-15.47-15.55
\end{aligned}
$$

Die Substanz stellt gelbe Nadeln dar, schmilzt bei $220^{\circ}$ und ist in Alkohol schwer, doch leichter als die $\alpha$-Verbindung löslich, in Eisessig löst sie sich viel leichter als diese.

$0.3133 g$ der bei $100^{\circ}$ getrockneten Substanz gaben $28.75 \mathrm{~cm}^{3}$ Stickstoff bei $743 \mathrm{~mm}$ Druck und $21^{\circ}$.

In 100 Theilen:

$$
N \ldots \ldots \frac{10 \cdot 20}{\text { Gefunden }} \quad \underbrace{\begin{array}{r}
\text { Berechnet fur } \\
\mathrm{C}_{13} \mathrm{H}_{6} \mathrm{O}\left(\mathrm{NO}_{2}\right)_{2}
\end{array}}_{10 \cdot 37}
$$

Das Keton wurde in Eisessig gelöst und Phenylhydrazin zugegeben. Schon beim ersten Tropfen trat Rothfärbung ein; bei weiterem Erwärmen schied sich sofort ein krystallinischer rother Niederschlag von ganz anderer Nuance aus, als dem Hydrazon der $\alpha$-Verbindung eigenthümlich ist. Abgesaugt und mit Eisessig und Alkohol gewaschen, hatten die nach dem Trocknen glitzernden Kryställchen eine dunkel kupferrothe Farbe und erwiesen sich unter dem Mikroskope als durchaus homogene, rechteckig begrenzte flache Nadeln. Aus Eisessig umkrystallisirt, schmelzen sie, rasch erhitzt, bei $227-228^{\circ}$ unter Gasentwicklung.

$0.1959 \mathrm{~g}$ bei $100^{\circ}$ getrockneter Substanz gaben $26.5 \mathrm{~cm}^{3}$ Stickstoff bei $740 \mathrm{~mm}$ Druck und $19^{\circ}$ 
In 100 Theilen:

$$
\mathrm{N} \ldots \ldots \ldots \underbrace{\text { Gefunden }}_{16 \cdot 05} \quad \underbrace{\begin{array}{c}
\text { Berechnet für } \\
\mathrm{C}_{19} \mathrm{H}_{12} \mathrm{~N}_{4} \mathrm{O}_{4}
\end{array}}_{15 \cdot 55}
$$

Es hat sich demnach ergeben, dass sämmtliche von uns untersuchte Chlor-, Brom- und Nitro-Substitutionsproducte des Fluorenons befähigt sind. mit grösster Leichtigkeit Hydrazone zu liefern. Von manchen der Substitutionsproducte ist die Parastellung der Substituenten zur Bindungsstelle der zwei Phenylgruppen erwiesen; von den anderen machen es die Versuchsresultate auf Grund bekannter anderseitiger Beobachtungen wahrscheinlich, dass sie nicht die Orthostellung zur Carbonylgruppe einnehmen.

Die bisherigen Beobachtungen sind daher nicht geeignet. Nateriale zurBeurtheilung des Einflusses der Natur und Stellung der Substituenten auf die Möglichkeit der Hydrazonbildung zu liefern. Die Untersuchung wird daher noch auf weitere Substitutionsproducte des Fluorenons ausgedehnt werden. Erst dann soll die Discussion dieser Frage, auch unter Heranziehung der über dieselbe bereits vorliegenden Beobachtungen erfolgen. 